Parallel Processing Letters

(C) World Scientific Publishing Company

\title{
PRAM MEMORY ALLOCATION AND INITIALIZATION
}

\author{
LISA HIGHAM \\ Department of Computer Science, University of Calgary \\ Calgary, Alberta T2N 4NC, Canada \\ and \\ ERIC SCHENK \\ Department of Computer Science, University of Toronto \\ Toronto, Ontario MSA 1S5, Canada \\ Received (received date) \\ Revised (revised date) \\ Communicated by (Name of Editor)
}

\begin{abstract}
Two useful and practical techniques for managing memory on a parallel random access machine (PRAM) are presented. One is a scheme for an $n / \log n$ processor EREW PRAM that dynamically allocates and deallocates at most $n$ records in $O(\log n)$ time. The other is a simulation of a PRAM with initialized memory by one with uninitialized memory. A CREW PRAM variant of the technique justifies the assumption that memory can be assumed to be appropriately initialized with no asymptotic increase in time but a factor of $n$ increase in space. An EREW PRAM solution incurs a factor of $O(\log n)$ increase in time but only a constant factor increase in space.
\end{abstract}

Keywords: Parallel Algorithms, PRAM, Memory Management, Initialization.

\section{Introduction}

Procedures for memory management are commonly assumed tools for algorithms that maintain dynamic data structures. Such tools have been thoroughly studied for sequential machines for decades. This paper presents two useful and practical techniques for managing memory on a parallel random access machines (PRAM). One is a scheme for an $n / \log n$ processor Exclusive Read Exclusive Write PRAM that dynamically allocates and deallocates memory to any subset of the processors in $\mathrm{O}(\log n)$ time. The other is a scheme for simulating PRAMs possessing initialized memory with corresponding PRAMs possessing uninitialized memory.

A PRAM is a common abstraction of a parallel machine that is useful for the design and analysis of parallel algorithms. It is a collection of synchronized independent sequential processors with unique identifiers and a shared global memory. In each time step, each processor can read a location in the global memory, perform a local computation, and then write to a location in the global memory. In an EREW (Exclusive Read Exclusive Write) PRAM no two processors may simultaneously access the same memory location for either reading or writing; in a CREW (Concurrent Read Exclusive Write) PRAM, simultaneous reading, but not writing, is permitted. (See Karp and Ramachandran [1] for an overview of PRAM results.)

PRAM algorithms that manipulate dynamic data structures need to be able to secure and release memory from the global store in parallel. For example, Paul, Vishkin and Wagener present algorithms [2] for maintaining 2-3-trees that depend upon dynamic allocation and deallocation of memory. An extension to general B-trees [3] similarly depends on parallel memory management. Section 2 describes a general scheme for an $n / \log n$ processor EREW PRAM that dynamically allocates and deallocates memory to any subset of the processors in $\mathrm{O}(\log n)$ time. 
When designing PRAM algorithms it is sometimes convenient to assume that all memory is appropriately initialized. For example, Schenk uses this assumption to detect whether a given memory cell has been written during the course of the computation [4]. Since it is conceivable that this assumption may make a problem easier to solve than it would otherwise be, it is important to determine its cost in terms of time, processors and memory. A technique from the folklore of computer science [5], simulates initialized memory with uninitialized memory for a sequential random access machine with only a constant factor increase in time and space. In section 3 we adapt this technique to the $n$ processor CREW PRAM setting with no asymptotic increase in time but a factor of $n$ increase in memory size, and to an EREW PRAM setting with a factor of $\mathrm{O}(\log n)$ increase in time and a constant factor increase in space.

\section{Memory Management}

This section describes algorithms and data structures that maintain PRAM memory in such a way that deallocated memory is captured and reused rather than new memory being consumed. We present algorithms for dynamically allocating and deallocating sets of $n$ or fewer records of a fixed size on an $n / \log n$ processor EREW PRAM in O(log $n)$ time.

\subsection{Data Structures}

The algorithms maintain all available memory in two data structures using an additional array $w_{0}, \ldots, w_{3 n}$ for working space. A contiguous array, mem $_{\text {start }}$, mem $_{\text {start }+1}, \ldots$ contains the memory that has never been allocated. A linked list of balanced binary trees called the free list contains deallocated records. The first tree in the free list contains at most $2 n$ records and if there are two or more trees then the first tree has at least $n$ records. All other trees in the list contain exactly $n$ records. Initially all available memory is in the array; that is, start is set to one and the free-list is empty.

We assume that the size of the record, say record-size, is large enough to contain two pointers, left and right, which are used to maintain the free list structure. One record in each tree is used to maintain the linked list of trees by having left point to the actual tree stored in the linked list, and right point to the next record in the linked list. Other records in the free list use left and right as usual to point to the roots of left and right subtrees. The variable head points to the head of the free list, and size counts the number of records in the tree at the head of the list (including the extra record that forms the linked list).

\subsection{Subroutines}

Two tree manipulation routines are required by the allocation algorithm: one constructs a balanced binary tree out of an array, the other maps a tree into an array.

Constructing a balanced binary tree out of the records pointed to by an array $w_{0}, \ldots, w_{m}$ is done by mapping element 1 onto the root, and elements $2 i$ and $2 i+1$ onto the children of $i$. Note that the first element of the array stores the link to the next tree in the free list.

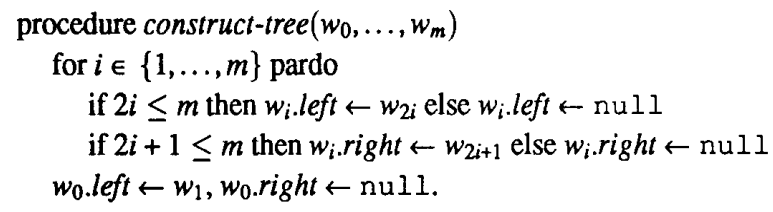

On an $n / \log n$ processor EREW PRAM, this pnocedure can be performed optimally in $\mathrm{O}(m \log n / n+\log n)$ time by assigning $\lceil m \log n / n\rceil$ iterations of the pardo loop to each processor. The extra $\log n$ term is required to broadcast the value of $m$ to all processors.

Mapping the nodes of a balanced binary tree of size $m$ onto an array $w_{0}, \ldots, w_{m}$ can be accomplished in $\mathrm{O}(m / p+$ $\log m$ ) time on a $p$ processor EREW PRAM as follows. First the root of the tree is mapped onto element $w_{1}$. Then, using $\lfloor\log p\rfloor$ iterations of a parallel loop, the top $\lfloor\log p\rfloor+1$ levels of the binary tree are mapped onto the array. The mapping process is completed by assigning a processor to each node at level $\lfloor\log p\rfloor+1$. Each processor then 
recursively maps the subtree found at its assigned node into the array. The node that maintains the tree's position in the linked list is mapped into element $w_{0}$ of the array.

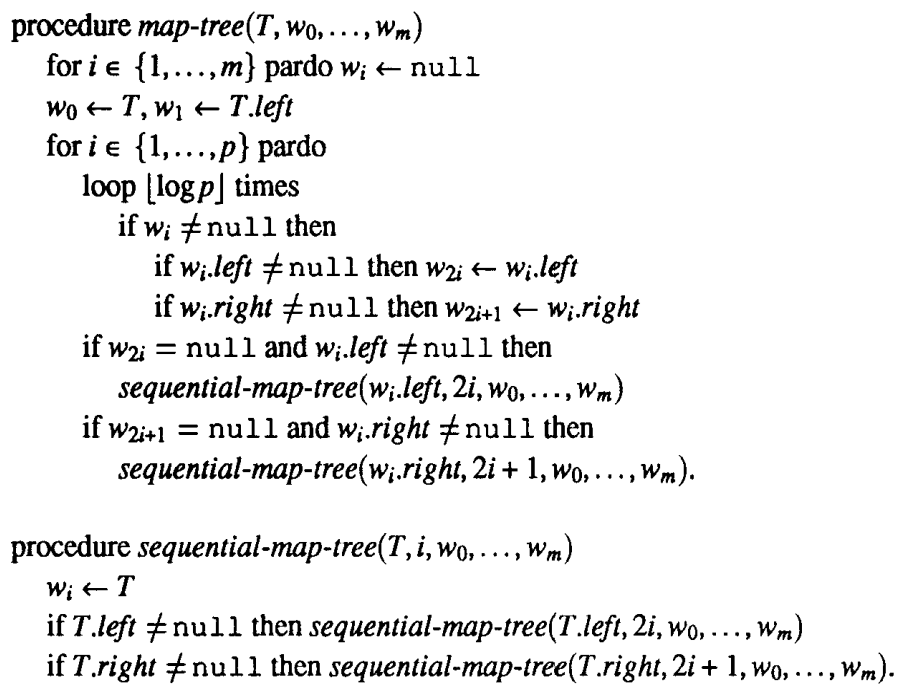

Setting $p$ to $n / \log n$ processors, this procedure takes $\mathrm{O}(m \log n / n+\log m)$ time.

\subsection{Allocation and Deallocation}

Allocation of $k \leq n$ records, if $k>$ size, is from the front of the array mem. Otherwise the $k$ required records are allocated from the tree at the head of the free list. If this tree contains fewer than $n$ records after $k$ have been removed, then it is merged with the next tree (if any) in the list. Using the procedures given in subsection 2.2 , this requires $\mathrm{O}(\log n)$ time on an $n / \log n$ processor EREW PRAM.

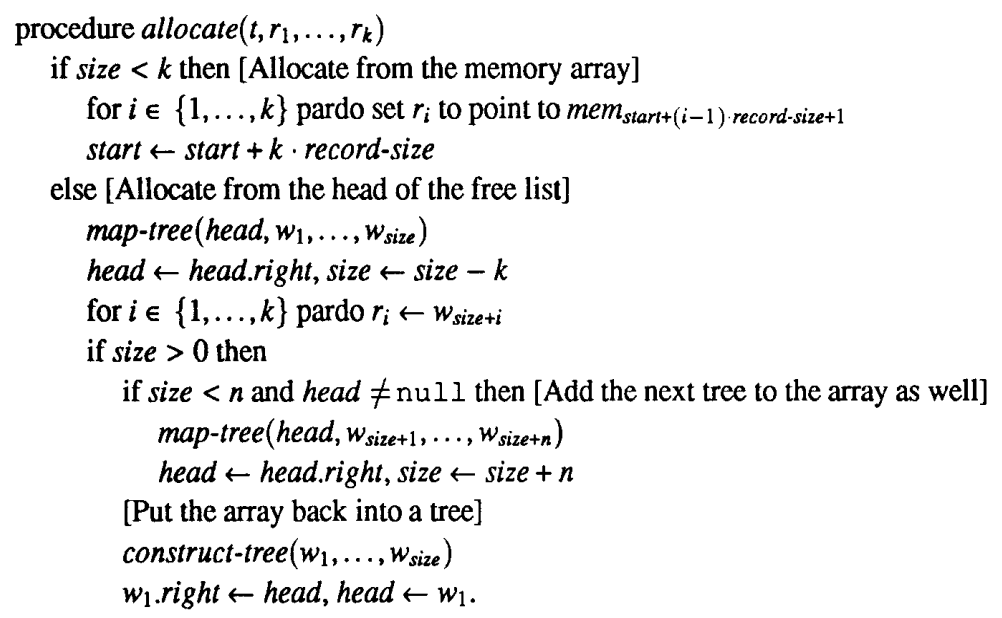

Deallocation of $k \leq n$ records is accomplished by adding them to the tree at the head of the free list. If the resulting tree has more than $2 n$ records, $n$ records are formed into a separate tree and inserted just after the head of the free list. Using the procedures given in subsection 2.2, this requires $\mathrm{O}(\log n)$ time on an $n / \log n$ processor EREW PRAM. 


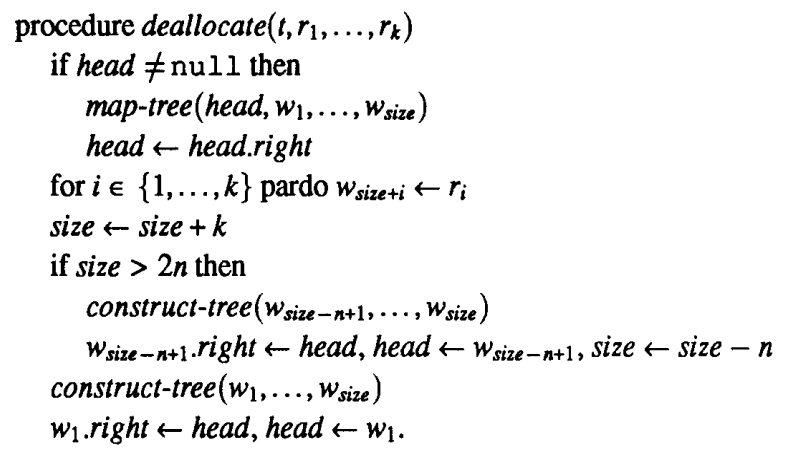

Taking these results together, we have the following theorem.

Theorem 1 For $k \leq n, k$ records of the same size, say s, can be allocated or deallocated in $\mathrm{O}(\log n)$ time on an $n / \log n$ processor EREW PRAM. Furthermore, for all times $t$, the total space used is $\left(A_{t}-D_{t}\right) s+\mathrm{O}(n)$, where $A_{t}$ (respectively $D_{t}$ ) is the total number of records allocated (respectively deallocated) at or before time $t$.

To manage different record sizes, separate free lists can be maintained for each size of record. It is not necessary to maintain more than one array mem. Allocations of each record size still require the cooperation of all $n / \log n$ processors.

By replacing the linked list of trees with a linked list of arrays, our algorithms can be transformed into constant time $n$ processor CREW PRAM algorithms for allocating and deallocating exactly $n$ records. This entails separately allocating arrays of size $n$ and allocating and deallocating them as the free list grows or shrinks. This increases the space usage by at most $m$. However, if fewer than $n$ records are allocated or deallocated, the time required by our CREW variants increases beyond constant time.

\section{Initialization}

This section describes two simulations of an $n$ processor PRAM with initialized memory by an $n$ processor PRAM with uninitialized memory. Let $\alpha$ be an algorithm for an $n$ processor EREW PRAM with initialized memory and let $t_{\alpha}(n)$ be the time complexity and $s_{\alpha}(n)$ be the space complexity of $\alpha$ on inputs of size $n$. The first simulation, $S(\alpha)$, simulates $\alpha$ on an $n$ processor EREW PRAM with uninitialized memory in time $\mathrm{O}\left(t_{\alpha}(n) \log n\right)$ and $3 s_{\alpha}(n)+1$ space. Let $\beta$ be an algorithm for an $n$ processor CREW PRAM with initialized memory and let $t_{\beta}(n)$ be the time complexity and $s_{\beta}(n)$ be the space complexity of $\beta$ on inputs of size $n$. The second simulation, $\mathrm{R}(\beta)$, simulates $\beta$ on an $n$ processor CREW PRAM with uninitialized memory in $\mathrm{O}\left(t_{\beta}(n)\right)$ time and $(n+3) s_{\beta}(n)$ space. Both simulations maintain a data structure in such a way that it is easy to distinguish between a value that has been written and uninitialized garbage.

\subsection{EREW PRAM simulation}

Denote the memory array of the $n$ processor EREW PRAM with initialized memory by $M$. On the $n$ processor EREW PRAM with uninitialized memory, create one extra integer variable, next-space, and $3 n$ cells of working space, and partition the remaining memory into three arrays, $A, B$ and $C$ by interleaving. Array $A$ is used to simulate memory $M$. Arrays $B$ and $C$ and the variable next-space are used to keep track of those positions of $M$ that have been written. Location $l$ of array $X$ is denoted $X[l]$.

Simulation $S$ maintains the following two part invariant for every location $l$ in $M$ : (1) $M[l]$ has been written by $\alpha$ if and only if $B[l] \in\{1, \ldots$, next-space -1$\}$ and $C[B[l]]=l$; and (2) if $M[l]$ has been written by $\alpha$ then $A[l]$ contains the last value written to $M[l]$.

Setting next-space to one ensures that the invariant holds initially. Suppose, at some time step of algorithm $\alpha$, processors $1, \ldots, n$ access memory locations $M\left[l_{1}\right], \ldots, M\left[l_{n}\right]$ respectively, and suppose the invariant holds before simulating this step. $\mathrm{S}$ has each processor $j$ first determine whether the $M\left[l_{j}\right]$ has already been written. If the memory access instruction is a read, then for each processor $j$, if $M\left[l_{j}\right]$ has been previously written then $j$ reads $A\left[l_{j}\right]$; otherwise $M\left[l_{j}\right]$ has not been written so $j$ assumes the initial value. If the memory access instruction is a write, then first those 
processors writing to memory locations that have not been previously written, cooperate to update arrays $B$ and $C$ and the value of next-space to ensure that the invariant is maintained. Then each processor $j$ writes to $A\left[l_{j}\right]$.

More specifically, define $P_{j}$ to be the predicate $\left(B\left[l_{j}\right] \in\{1, \ldots\right.$, next-space -1$\left.\} \wedge C\left[B\left[l_{j}\right]\right]=l_{j}\right)$. According to the invariant, $M\left[l_{j}\right]$ has been written if and only if $P_{j}$. Let write-check $\left(l_{1}, \ldots, l_{n}, x_{1}, \ldots, x_{n}, y_{1}, \ldots, y_{n}\right)$ be a procedure that for $j \in\{1, \ldots, n\}$ sets $x_{j}$ to $P_{j}$ and sets $y_{j}$ to the size of the set $\left\{i \leq j: \neg P_{i}\right\}$.

Given write-check, the following procedures perform, respectively, one parallel read and write step of the simulation S. Procedure read sets $v_{j}$ to the value in $M\left[l_{j}\right]$. Procedure write sets $A\left[l_{j}\right]$, which simulates $M\left[l_{j}\right]$, to the value $v_{j}$.

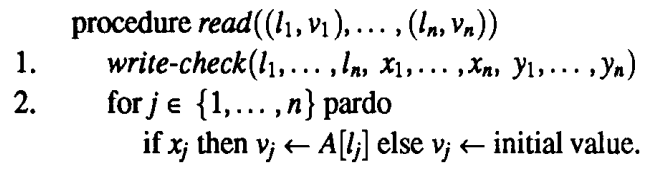

1. write-check $\left(l_{1}, \ldots, l_{n}, x_{1}, \ldots, x_{n}, y_{1}, \ldots, y_{n}\right)$

2. for $j \in\{1, \ldots, n\}$ pardo

$$
\begin{aligned}
& \text { if } \neg x_{j} \text { then } \\
& \quad B\left[l_{j}\right] \leftarrow \text { next-space }+y_{j}-1, C\left[\text { next-space }+y_{j}-1\right] \leftarrow l_{j} \\
& A\left[l_{j}\right] \leftarrow v_{j}
\end{aligned}
$$

3. next-space $\leftarrow$ next-space $+y_{n}$.

It is easily checked from the pseudo-code that the invariant holds after read or write provided that it held before this step. Clearly step two of read and steps two and three of write complete in constant time on an $n$ processor EREW PRAM.

It remains to provide more details for write-check. The values $y_{1}$ through $y_{n}$ can be computed from $x_{1}$ through $x_{n}$ in $\mathrm{O}(\log n)$ time on an EREW PRAM using Prefix Sums [1]. Therefore we need only to determine how to compute $x_{1}, \ldots, x_{n}$ (that is $P_{1}, \ldots, P_{n}$ ). Even though all $l_{j}$ 's are distinct, processors might read nondistinct positions in array $C$. Also, each processor needs to access next-space. Some care is needed to ensure exclusive access in the simulation. First all processors cooperate to lexicographically sort the pairs $\left(B\left[l_{1}\right], 1\right), \ldots,\left(B\left[l_{n}\right], n\right)$, yielding $D_{1}, \ldots, D_{n}$ where $D_{j}=\left(B\left[l_{k}\right], k\right)$, for some $k \in\{i, \ldots, n\}$. Let $D_{j, 1}$ (respectively $D_{j, 2}$ ) denote the first (respectively second) coordinate of $D_{j}$. The processors then determine all maximal intervals $[i \ldots k]$ such that $D_{i, 1}=D_{k, 1}$. For each such interval $[i \ldots k]$, processor $i$ reads $C\left[D_{i, 1}\right]$ and initiates a broadcast of the result to the remaining processors in its interval. Next a broadcast is used to distribute the value of next-space to each processor. Each processor $j$ can now determine whether $D_{j, 1} \in\{1, \ldots$, next-space -1$\}$ and whether $C\left[D_{j, 1}\right]=l_{D_{j 2}}$ without conflict. If either test fails, then $x_{D_{j 2}}$ is set to false, otherwise $x_{D_{j 2}}$ is set to $t$ rue. Broadcast is a basic technique that can be achieved on an $n$ processor EREW PRAM in $\mathrm{O}(\log n)$ time [6]. Cole's parallel merge sort [7] can be used to sort in $\mathrm{O}(\log n)$ time. All other operations requires just constant time.

Theorem 2 Let $\alpha$ be an algorithmfor an n processor EREW PRAM with initialized memory taking $t_{\alpha}(n)$ time and using $s_{\alpha}(n)$ space. Then $S(\alpha)$ simulates $\alpha$ on an $n$ processor EREW PRAM with uninitialized memory in time $\mathrm{O}\left(t_{\alpha}(n) \log n\right)$ and space $3 s_{\alpha}(n)+3 n+1$.

\subsection{CREW PRAM simulation}

In the EREW PRAM simulation, the function write-check uses $\mathrm{O}(\log n)$ time in order to avoid read collisions of the variable next-space and (possibly) of locations in array $C$. If concurrent reads are permitted then determining $P_{1}$ through $P_{n}$ can be achieved in constant time with no change in data structures. In step two of procedure write however, each processor writing to a new location needs to be allocated a new distinct record from the single array $C$ and in step three the pointer next-space must be incremented by the number of such processors. Determining this number requires more than constant time even with concurrent reads. To circumvent this problem, we replace array $C$ and variable next-space with a separate array $C_{j}$ and variable next-space ${ }_{j}$ for each $j \in\{1, \ldots, n\}$. This ensures that in each step, at most one record will be allocated from any one array $C_{j}$ and that each next-space $e_{j}$ is incremented by at most 1 . 
Specifically, on the $n$ processor CREW PRAM with uninitialized memory, create $n$ extra integer variables, next-space for $_{j} \in\{1, \ldots, n\}$, and $3 n$ cells of working space, and partition the remaining memory into $n+2$ arrays, $A, B$ and $C_{j}$ for $j \in\{1, \ldots, n\}$ by interleaving. Array $A$ is used to simulate the memory, say $M$, of the $n$ processor CREW PRAM with initialized memory. Entries in $B$ are now a pair $(p, i)$ where $p$ is an integer between 1 and $n$ and $i$ is an index into array $C_{p}$.

Simulation R maintains the following two part invariant for every location $l$ in $M$ : (1) $M[l]$ has been written by $\beta$ if and only if $B[l]=(p, i)$ where $p \in\{1, \ldots, n\}$ and $i \in\left\{1, \ldots\right.$, next-space $\left.e_{p}-1\right\}$ and $C_{p}[i]=l$; and $(2)$ if $M[l]$ has been written by $\beta$ then $A[l]$ contains the last value written to $M[l]$.

To initialize the structure, each processor $j$ sets next-space $e_{j}$ to 1 thus ensuring that the invariant holds initially. Suppose, as previously, at some time step of algorithm $\beta$, processors $1, \ldots, n$ access memory locations $M\left[l_{1}\right], \ldots, M\left[l_{n}\right]$ respectively, and suppose the invariant holds before simulating this step. Let $P_{j}$ be the predicate $\left(B\left[l_{j}\right]=(p, i) \wedge p \in\right.$ $\{1, \ldots, n\} \wedge i \in\{1, \ldots$, next-space $\left.p-1\} \wedge C_{p}[i]=l_{j}\right)$. Let write-check $^{\prime}\left(l_{1}, \ldots, l_{n}, x_{1}, \ldots, x_{n}\right)$ be a procedure that sets $x_{j}$ to $P_{j}$ for $j \in\{1, \ldots, n\}$. Notice that since the model permits concurrent reads, write-check $k^{\prime}$ takes only constant time by having each processor $j$ compute $P_{j}$ independently.

A CREW PRAM read procedure is obtained from the procedure read by replacing write-check with write-check'. One parallel write step of the simulation $\mathrm{R}$ is as follows. For each $j \in\{1, \ldots, n\}, v_{j}$ is the value to be written to $A\left[l_{j}\right]$, which simulates $M\left[l_{j}\right]$.

1. write-check $\left(l_{1}, \ldots, l_{n}, x_{1}, \ldots, x_{n}\right)$.

2. for $j \in\{1, \ldots, n\}$ pardo

$$
\begin{aligned}
& \text { if } \neg x_{j} \text {, then } \\
& \quad B\left[l_{j}\right] \leftarrow\left(j, \text { next }_{\text {space }}\right), C_{j}\left[\text { next-space }_{j}\right] \leftarrow l_{j} . \\
& \quad n e x t-\text { space }_{j} \leftarrow \text { next-space } \\
& j
\end{aligned}
$$

It is easily checked that the invariant holds after crew-write provided it held immediately before its execution. Since the model is exclusive write, all $l_{j}$ are distinct and thus step two of crew-write takes constant time on a CREW PRAM.

Theorem 3 Let $\beta$ be an algorithm for an n processor CREW PRAM with initialized memory taking $t_{\beta}(n)$ time and using $s_{\beta}(n)$ space. Then $R(\beta)$ simulates $\beta$ on an $n$ processor CREW PRAM with uninitialized memory in time $\mathrm{O}\left(t_{\beta}(n)\right)$ and space $(n+3) s_{\beta}(n)+3 n$.

\section{Discussion and Open Problems}

In our simulations, each instruction executed by the PRAM with initialized memory is emulated by a short sequence of instructions on the PRAM with uninitialized memory. Of course, for any memory cell not written during the run of the algorithm, the contents of the original and simulated memories may differ. To extend the simulations so that the final outputs are the same, we have to assume that the final output is actually written by the PRAM processors rather than just being declared as some arbitrary part of the contents of memory.

According to simulation $\mathrm{R}$, it is justifiable to assume that memory is preinitialized when determining a problem's time complexity on a CREW PRAM. We do not know if such an assumption can be justified for an EREW PRAM. Furthermore, we do not know how to justify the assumption for a CREW PRAM without increasing the size of memory by a linear factor.

\section{Acknowledgements}

This research was supported in part by a research grant and a postgraduate scholarship, both from the Natural Sciences and Engineering Research Council of Canada. 


\section{References}

1. Richard M. Karp and Vijaya Ramachandran. A survey of parallel algorithms for shared-memory machines. In J.van Leeuwen, editor, Handbook of Theoretical Computer Science, volume A, chapter 17. Elsevier Science Publishers, Amsterdam, The Netherlands, and The MIT Press, Cambridge, Massachusetts, U.S.A., 1990.

2. W. Paul, U. Vishkin, and H. Wagener. Parallel dictionaries on 2-3 trees. In Lecture Notes in Computer Science 143: Proceedings of the 10th Colloquium on Automata, Languages and Programming, pages 597-609. Springer Verlag, 1983.

3. Lisa Higham and Eric Schenk. Maintaining B-trees on an EREW PRAM. Technical Report 91/446/30, Department of Computer Science, University of Calgary, September 1991. Submitted for publication.

4. Eric Schenk. The parallel asynchronous recursion model. Master's thesis, Department of Computer Science, University of Calgary, Canada, 1992. Research Report No. 92/473/11.

5. K. Abrahamson. private communication.

6. Alan Gibbons and Wojciech Rytter. Efficient Parallel Algorithms. Cambridge University Press, Cambridge, Great Britain, 1988.

7. Richard Cole. Parallel merge sort. SIAM J. Comput., 17(4):770-785, August 1988. 